\title{
Rivlin-Ericksen Fluid in Tube of Varying Cross-section with Mass and Heat Transfer
}

\author{
Nabil T. El Dabe, Galal M. Moatimid, and Hoda S. M. Ali \\ Department of Mathematics, Faculty of Education, Ain Shams University, Heliopolis, Cairo, Egypt \\ Reprint requests to Dr. G. M. M.; E-mail: gal_moa@hotmail.com
}

Z. Naturforsch. 57 a, 863-873 (2002); received April 28, 2002

In this paper, the problem of heat and mass transfer due to the steady motion of a RivlinEricksen fluid in tubes of varying cross-section is considered. Suction at tube walls is taken into account. Under the assumption that the deformations of the boundaries are small, the equations of motion have been solved by using a perturbation technique. The temperature and concentration distributions are obtained. The effects of various physical parameters are discussed. The Nusselt and Sherwood numbers are obtained. A set of figures for a quantitative illustration is presented.

Key words: Non-Newtonian Fluids; Heat Transfer; Mass Transfer.

\section{Introduction}

The study of the influence of mass and heat transfer on Newtonian and non-Newtonian fluids has become important in the last few years. This importance is due to a number of industrial processes. Examples are food processing, biochemical operations and transport in polymers. Flowing over deformable boundaries has also gained importance because of its immediate practical application in lubrication technology, biophysical flows and many other transportation types. Besides, the characteristics of the flow of blood through arteries and veins are of considerable medical interest.

In the past, there has been a number of studies of non-Newtonian heat and mass transfer in tubes. Mathur and Bhatnagar [1] have studied the steady axial flow of a Rivlin-Ericksen fluid in a wavy annulus with heat transfer. They found that the non-Newtonian parameters affect the velocity field, the stresses and the temperature field. They also found that the streamlines near the boundaries run parallel to them and there is no change in their deformity as a result of the no slip conditions. O'Neill [2] studied the effects produced by small-scale plane or axisymmetrical boundary irregularities on steady parallel flows of incompressible viscous fluids. He considered the flow between two parallel planes as well as that in a cylindrical pipe of circular cross-section. Under the restriction that the Reynolds number of the perturbed flow is small enough, he obtained the solution of the stream function. Srivastava [3] has studied the same problem for a special class of Rivlin-Ericksen "second order" fluids. He obtained the velocity components and the pressure in powers of the oscillation amplitude of the plate. He considered the flow of an incompressible second-order fluid due to torsional oscillations of an infinite plate when the fluid is infinite in extent as well as the case when it is bounded by another stationary parallel plate.

Kawase and Ulbrecht [4] investigated the heat and mass transfer in a non-Newtonian fluid flow with different velocity profiles. They considered a laminar as well as a turbulent non-Newtonian fluid. They found reasonable agreement between their results and the available experimental data. Dalal and Mazumder [5] revealed that for a viscoelastic fluid, the dispersion coefficient changes cyclically with a double frequency period and reaches asymptotically a stationary state after a certain time as in the case of a Newtonian fluid and its increase with the viscoelastic parameter. Pereira et al. [6] found the temperature distribution of non-Newtonian fluids in a laminar flow heated in a double tube heat exchanger, and compared the predictions by analytical and numerical methods. Hung and Perng [7] discussed the flow of a non-Newtonian fluid in the entrance region of a tube with porous walls. They solved the modified Navier-stockes equations numerically. Their analysis resulted in velocity distributions, pressure drops, and skin-friction coef- 
ficients in the cases of blowing and suction. Away from the entrance, their results agree well with previous works. Parikh and Mahalingam [8] obtained analytical solutions for the wall temperature profile of a power law fluid in laminar flow in a circular tube. Their computed results are compared with measured values obtained from a horizontal recirculating flow experimental unit. Chandra and Prasad [9] studied the steady flow of an incompressible fluid in a rigid tube of slowly varying cross-section with absorbing walls. They found the effect of fluid absorption through a permeable wall by prescribing the flux as an arbitrary function of the axial distance. They obtained the low Reynolds number flow and the expressions for various flow characteristics by using perturbation analysis. Ahmed and Attia [10] discussed the laminar flow with heat transfer of an incompressible, electrically conducting non-Newtonian fluid in an eccentric annulus in the presence of an external uniform magnetic field. They found a numerical solution of the governing differential equations. Also, they obtained the influence of the magnetic field on both the velocity and temperature distributions. Kurdyumov and Linan [11] studied the steady, two dimensional, free convection around line sources of heat and heated cylinders in unbounded saturated porous media. Their study is based on the Boussinesq equations, with the velocities calculated using Darcy's law.

Prasad and Chandra [12] have considered the problem of a visco-elastic fluid in tubes of varying crosssection with suction or injection. Their analysis ignored the presence of mass and heat transfer. Because of the wide range of practical importance of the mass and heat transfer, the present paper considered the mass and heat transfer of a steady slow motion of a Rivlin-Ericksen fluid in tubes of varying cross-section with suction. Analytical solutions for the temperature and the concentration distributions are obtained. The analysis is based on a perturbation technique. The rate of the mass and heat transfer is also obtained. The main idea of the present work was to mathematically model these phenomena, and the other purpose was to find the relation between the different parameters and the external forces. Also, our aim was to show how the temperature and concentration may be controlled by changing the various relevant physical parameters.

\section{Formulation of the Problem}

Consider the steady incompressible flow of a Rivlin-Ericksen fluid in a tube of slowly varying cir- cular cross-section and having a permeable wall. We choose cylindrical polar coordinates $(X, \theta, R)$. The radius $R=a(X)$ of the tube is an arbitrary function of $X$. Considering a swirl free flow with the velocity vector $\boldsymbol{V}=(U, V, 0)$ in an $(X, R, \theta)$ coordinate system, the equations governing the steady flow of an incompressible fluid reduce to

$$
\begin{gathered}
\rho\left(U \frac{\partial U}{\partial X}+V \frac{\partial U}{\partial R}\right)=-\frac{\partial P}{\partial X}+\frac{\partial \tau_{R X}}{\partial R}+\frac{\tau_{R X}}{R}+\frac{\partial \tau_{X X}}{\partial X} \\
\rho\left(U \frac{\partial V}{\partial X}+V \frac{\partial V}{\partial R}\right)=-\frac{\partial P}{\partial R}+\frac{\partial \tau_{R R}}{\partial R} \\
+\frac{\tau_{R R}-\tau_{\theta \theta}}{R}+\frac{\partial \tau_{R X}}{\partial X} \\
\frac{\partial U}{\partial X}+\frac{\partial V}{\partial R}+\frac{V}{R}=0 \\
\left.U \frac{\partial T}{\partial X}+V \frac{\partial T}{\partial R}\right)=k\left(\frac{\partial^{2} T}{\partial R^{2}}+\frac{1}{R} \frac{\partial T}{\partial R}+\frac{\partial^{2} T}{\partial X^{2}}\right)+\phi \\
U \frac{\partial C}{\partial X}+V \frac{\partial C}{\partial R}=D\left(\frac{\partial^{2} C}{\partial R^{2}}+\frac{1}{R} \frac{\partial C}{\partial R}+\frac{\partial^{2} C}{\partial X^{2}}\right) \\
+D \frac{k_{T}}{T_{m}}\left(\frac{\partial^{2} T}{\partial R^{2}}+\frac{1}{R} \frac{\partial T}{\partial R}+\frac{\partial^{2} T}{\partial X^{2}}\right)
\end{gathered}
$$

where $\phi$ (the dissipation function $)=\tau_{i j} \partial v_{i} / \partial X_{j}, \rho$ is the constant fluid density, $P$ the pressure, $C$ the concentration, $T$ the temperature, $c_{p}$ the specific heat at constant pressure, $D$ the coefficient of mass diffusivity, $T_{m}$ the mean fluid temperature, $k_{T}$ the thermal diffusion ratio and $k$ the thermal conductivity. The stress components $\tau_{R R}, \tau_{R X}, \tau_{X X}$ and $\tau_{\theta \theta}$ are given by the constitutive equations

$$
\begin{gathered}
\tau_{i j}=\mu_{1} d_{i j}+\mu_{2} E_{i j}+\mu_{3} d_{i}^{\alpha} d_{\alpha j}, \\
d_{i j}=v_{, j}^{i}+v_{, i}^{j} \\
E_{i j}=a_{, j}^{i}+a_{, i}^{j}+2 v_{, i}^{m} v_{m, j}, \\
a_{i} \text { (acceleration vector) }=\frac{\partial v_{i}}{\partial t}+v^{m} v_{i . m},
\end{gathered}
$$

where $\tau_{i j}$ are the stress components, $v^{i}$ and $a^{i}$ being the velocity and acceleration components, and "," stands for covariant differentiation. $\mu_{1}, \mu_{2}$ and $\mu_{3}$ are the viscosity, elastico-viscosity and cross-viscosity coefficients of the fluid, respectively. 
The tube radius varies slowly, such that

$$
a(X)=a_{0} S\left(\frac{\varepsilon X}{a_{0}}\right), \varepsilon=\frac{a_{0}}{L} \ll 1 ; S(0)=1,
$$

where $a_{0}$ is the initial tube radius cross-section, $\varepsilon$ the wall variation parameter and $L$ the characteristic length of the tube [12].

\section{Boundary Conditions}

At the wall of the tube, the fluid velocity satisfies the conditions

$$
\left.\begin{array}{l}
\boldsymbol{V} \cdot \hat{t}_{w}=0 \\
\boldsymbol{V} \cdot \hat{n}_{w}=V_{w}
\end{array}\right\} \text { at } R=a(X)
$$

where $\hat{t}_{w}$ and $\hat{n}_{w}$ are the unit tangent and normal vectors at the tube wall, respectively, and $V_{w}$ is the constant suction velocity at the wall. The axial symmetry condition requires

$$
\begin{gathered}
\frac{\partial U}{\partial R}=0 \text { and } V=0 \text { at } R=0, \\
\frac{\partial T}{\partial R}=0 \text { and } \frac{\partial C}{\partial R}=0 \text { at } R=0, \\
T=T_{1} \text { and } C=C_{0} \text { at } R=a(X),
\end{gathered}
$$

where $C_{0}$ is the uniform concentration at the wall and $T_{1}$ is a uniform temperature at the wall.

$$
P=P_{\text {in }} \text { at } X=0, P=P_{\text {out }} \text { at } X=L .
$$

\section{Analysis}

We introduce the following dimensionless variables:

$$
\begin{gathered}
x=\frac{\varepsilon X}{a_{0}}, r=\frac{R}{a_{0}}, u=\frac{U}{U_{0}}, v=\frac{V}{\varepsilon U_{0}}, k_{1}=\frac{\mu_{2}}{\rho a_{0}^{2}} \\
k_{2}=\frac{\mu_{3}}{\rho a_{0}^{2}}, T_{i j}=\frac{a_{0}}{\mu_{1} U_{0}} \tau_{i j}, P=\frac{\varepsilon a_{0}}{\mu_{1} U_{0}} P, d=\frac{a_{0} U_{0}}{D}, \\
\operatorname{Re}=\frac{\rho a_{0} U_{0}}{\mu_{1}} \text { (Reynolds number), } \theta=\frac{T}{T_{1}}, \phi=\frac{C}{C_{0}}, \\
\operatorname{Pr}=\frac{\mu c_{p}}{k} \text { (Prandtl number), } \\
\text { Ec }=\frac{U_{0}^{2}}{c_{p} T_{1}} \text { (Eckert number), }
\end{gathered}
$$

$$
\begin{gathered}
\mathrm{Sc}=\frac{\mu_{1}}{D \rho}(\text { Schmidt number }), \\
\left.\mathrm{Sr}=\frac{\rho D T_{1} k_{T}}{\mu_{1} C_{0} T_{m}} \text { (Soret number }\right),
\end{gathered}
$$

where $U_{0}$ is the characteristic velocity.

Equations (1 - 5) and the boundary conditions (10 - 14) in dimensionless form become

(i) the equations of motion

$$
\begin{gathered}
\varepsilon \operatorname{Re}\left(u \frac{\partial u}{\partial x}+v \frac{\partial u}{\partial r}\right)=-\frac{\partial P}{\partial x}+\left(\frac{\partial}{\partial r}+\frac{1}{r}\right)\left(\varepsilon^{2} \frac{\partial v}{\partial x}+\frac{\partial u}{\partial r}\right) \\
+\operatorname{Re} \varepsilon k_{1}\left(\frac{\partial}{\partial r}+\frac{1}{r}\right)\left(\varepsilon^{2} \frac{\partial}{\partial x}\left(v \frac{\partial v}{\partial r}+u \frac{\partial v}{\partial x}\right)\right. \\
\left.+\frac{\partial}{\partial r}\left(u \frac{\partial u}{\partial x}+v \frac{\partial u}{\partial r}\right)+2 \varepsilon^{2} \frac{\partial v}{\partial r} \frac{\partial v}{\partial x}+2 \frac{\partial u}{\partial r} \frac{\partial u}{\partial x}\right) \\
+2 \varepsilon \operatorname{Re} k_{2}\left(\frac{\partial}{\partial r}+\frac{1}{r}\right)\left(\varepsilon^{2} \frac{\partial v}{\partial x}+\frac{\partial u}{\partial r}\right)\left(\frac{\partial v}{\partial r}+\frac{\partial u}{\partial x}\right) \\
+2 \varepsilon^{2} \frac{\partial^{2} u}{\partial x^{2}}+2 \varepsilon^{3} \operatorname{Re} k_{1} \frac{\partial}{\partial x}\left(\varepsilon \frac { \partial } { \partial x } \left(v \frac{\partial v}{\partial r}\right.\right. \\
\left.\left.+u \frac{\partial v}{\partial x}\right)+\varepsilon^{2}\left(\frac{\partial v}{\partial x}\right)^{2}+\left(\frac{\partial u}{\partial x}\right)^{2}\right) \\
+\operatorname{Re} \varepsilon k_{2} \frac{\partial}{\partial x}\left(4 \varepsilon^{2}\left(\frac{\partial u}{\partial x}\right)^{2}+\left(\varepsilon^{2} \frac{\partial v}{\partial x}+\frac{\partial u}{\partial r}\right)^{2}\right),(16)
\end{gathered}
$$$$
\varepsilon^{3} \operatorname{Re}\left(u \frac{\partial v}{\partial x}+v \frac{\partial v}{\partial r}\right)=-\frac{\partial P}{\partial r}+2 \varepsilon^{2}\left(\frac{\partial}{\partial r}+\frac{1}{r}\right) \frac{\partial v}{\partial r}
$$

$+2 \operatorname{Re} k_{1} \varepsilon\left(\frac{\partial}{\partial r}+\frac{1}{r}\right)\left(\varepsilon^{2} \frac{\partial}{\partial r}\left(v \frac{\partial v}{\partial r}+u \frac{\partial v}{\partial r}\right)\right.$

$$
\left.+\varepsilon^{2}\left(\frac{\partial v}{\partial r}\right)^{2}+\left(\frac{\partial u}{\partial r}\right)^{2}\right)
$$

$+\operatorname{Re}_{2} \varepsilon\left(\frac{\partial}{\partial r}+\frac{1}{r}\right)\left(4 \varepsilon^{2}\left(\frac{\partial v}{\partial r}\right)^{2}+\left(\varepsilon^{2} \frac{\partial v}{\partial x}+\frac{\partial u}{\partial r}\right)^{2}\right)$

$-2 \varepsilon^{2} \frac{v}{r^{2}}-2 \operatorname{Re} k_{1} \varepsilon^{3}\left(\frac{1}{r^{2}}\left(v \frac{\partial v}{\partial r}+u \frac{\partial v}{\partial x}\right)+\frac{v^{2}}{r^{3}}\right)$

$-4 \operatorname{Re} k_{2} \varepsilon^{3} \frac{v^{2}}{r^{3}}+\varepsilon^{2} \frac{\partial}{\partial x}\left(\frac{\partial v}{\partial x}+\frac{\partial u}{\partial r}\right)$ 
$+\operatorname{Re} k_{1} \varepsilon^{3} \frac{\partial}{\partial x}\left(\varepsilon^{3} \frac{\partial}{\partial x}\left(u \frac{\partial v}{\partial x}+v \frac{\partial v}{\partial r}\right)\right.$

$$
\left.+\frac{\partial}{\partial r}\left(v \frac{\partial u}{\partial r}+u \frac{\partial u}{\partial x}\right)+2 \varepsilon^{2} \frac{\partial v}{\partial r} \frac{\partial v}{\partial x}+2 \frac{\partial u}{\partial r} \frac{\partial u}{\partial x}\right)
$$

$+2 \operatorname{Re} k_{2} \varepsilon^{3} \frac{\partial}{\partial x}\left(\varepsilon^{2} \frac{\partial v}{\partial x}+\frac{\partial u}{\partial r}\right)\left(\frac{\partial v}{\partial r}+\frac{\partial u}{\partial x}\right)$,

(ii) the continuity equation

$$
\frac{\partial u}{\partial x}+\frac{\partial v}{\partial r}+\frac{v}{r}=0
$$

(iii) the energy equation

$$
\begin{aligned}
& \operatorname{Re} \varepsilon\left(u \frac{\partial \theta}{\partial x}+v \frac{\partial \theta}{\partial r}\right)=\frac{1}{\operatorname{Pr}}\left(\frac{\partial^{2} \theta}{\partial r^{2}}+\frac{1}{r} \frac{\partial \theta}{\partial r}+\varepsilon^{2} \frac{\partial^{2} \theta}{\partial x^{2}}\right) \\
& +\operatorname{Ec}\left(2 \varepsilon^{2}\left(\frac{\partial v}{\partial r}\right)^{2}+2 \varepsilon^{2}\left(\frac{v}{r}\right)^{2}+2 \varepsilon^{2}\left(\frac{\partial u}{\partial x}\right)^{2}\right. \\
& \left.+\left(\varepsilon^{2} \frac{\partial v}{\partial x}+\frac{\partial u}{\partial r}\right)^{2}\right)+\varepsilon \operatorname{ReEc} k_{1}\left[\varepsilon ^ { 2 } \left(2 \frac{\partial v}{\partial r} \frac{\partial}{\partial r}+2 \frac{v}{r^{2}}\right.\right. \\
& \left.+\varepsilon^{2} \frac{\partial v}{\partial x} \frac{\partial}{\partial x}+\frac{\partial u}{\partial r} \frac{\partial}{\partial x}\right)\left(v \frac{\partial v}{\partial r}+u \frac{\partial v}{\partial x}\right)+\left(2 \varepsilon^{2} \frac{\partial u}{\partial x} \frac{\partial}{\partial x}\right. \\
& \left.+\varepsilon^{2} \frac{\partial v}{\partial x} \frac{\partial}{\partial r}+\frac{\partial u}{\partial r} \frac{\partial}{\partial r}\right)\left(v \frac{\partial u}{\partial r}+u \frac{\partial u}{\partial x}\right)+2 \varepsilon^{2}\left(\frac{\partial v}{\partial r}\right)^{3} \\
& +2 \varepsilon^{2}\left(\frac{v}{r}\right)^{3}+2 \varepsilon^{2}\left(\frac{\partial u}{\partial x}\right)^{3}+2\left(\frac{\partial v}{\partial r}+\frac{\partial u}{\partial x}\right)\left(\left(\frac{\partial u}{\partial r}\right)^{2}\right. \\
& \left.\left.+\varepsilon^{2} \frac{\partial v}{\partial x} \frac{\partial u}{\partial r}+\varepsilon^{4}\left(\frac{\partial v}{\partial x}\right)^{2}\right)\right]
\end{aligned}
$$

$+\operatorname{ReEc} k_{2} \varepsilon\left(4 \varepsilon^{2}\left(\frac{\partial v}{\partial r}\right)^{3}+4 \varepsilon^{2}\left(\frac{v}{r}\right)^{3}+4 \varepsilon^{2}\left(\frac{\partial u}{\partial x}\right)^{3}\right.$

$$
\left.+3\left(\frac{\partial v}{\partial r}+\frac{\partial u}{\partial x}\right)\left(\varepsilon^{2} \frac{\partial v}{\partial x}+\frac{\partial u}{\partial r}\right)^{2}\right)
$$

(iv) the concentration equation

$$
\begin{aligned}
\varepsilon d\left(u \frac{\partial \phi}{\partial x}+v \frac{\partial \phi}{\partial r}\right)= & \frac{\partial^{2} \phi}{\partial r^{2}}+\frac{1}{r} \frac{\partial \phi}{\partial r}+\varepsilon^{2} \frac{\partial^{2} \phi}{\partial x^{2}} \\
& +\operatorname{ScSr}\left(\frac{\partial^{2} \theta}{\partial r^{2}}+\frac{1}{r} \frac{\partial \theta}{\partial r}+\varepsilon^{2} \frac{\partial^{2} \theta}{\partial x^{2}}\right)
\end{aligned}
$$

The boundary conditions become

$$
\begin{gathered}
u+\varepsilon^{2} v \frac{d S(x)}{d x}=0 \text { at } r=S(x) \\
v-u \frac{\mathrm{d} S(x)}{\mathrm{d} x}=v_{w}\left[1+\varepsilon^{2}\left(\frac{\mathrm{d} S}{\mathrm{~d} x}\right)^{2}\right]^{1 / 2} \text { at } r=S(x) \\
\frac{\partial u}{\partial r}=0 \text { and } v=0 \text { and } r=0 \\
\frac{\partial \theta}{\partial r}=0 \text { and } \frac{\partial \phi}{\partial r}=0 \text { and } r=0 \\
\theta=\phi=1 \text { at } r=S(x) \\
P=P_{\text {in }} \text { at } x=0 \text { and } P=P_{\text {out }} \text { at } x=1
\end{gathered}
$$

\section{Method of Solution}

We seek the solution of $(16-26)$ in terms of the small perturbation parameter $\varepsilon$ in the form

$$
\begin{aligned}
(u, v, P, \theta, \phi)= & \left(u^{(0)}, v^{(0)}, P^{(0)}, \theta^{(0)}, \phi^{(0)}\right) \\
& +\varepsilon\left(u^{(1)}, v^{(1)}, P^{(1)}, \theta^{(1)}, \phi^{(1)}\right)+o\left(\varepsilon^{2}\right) .
\end{aligned}
$$

The velocity components $u^{(0)}$ and $v^{(0)}$ are found by [12] as

$$
\begin{gathered}
u^{(0)}=\frac{1}{4} \frac{\mathrm{d} P^{(0)}}{\mathrm{d} x}\left(r^{2}-S^{2}\right), \\
v^{(0)}=\frac{r}{16}\left[\frac{\mathrm{d}^{2} P^{(0)}}{\mathrm{d} x^{2}}\left(2 S^{2}-r^{2}\right)+4 S \frac{\mathrm{d} S}{\mathrm{~d} x} \frac{\mathrm{d} P^{(0)}}{\mathrm{d} x}\right],
\end{gathered}
$$

where

$$
\frac{\mathrm{d} P^{(0)}}{\mathrm{d} x}=\frac{16 v_{w}}{S^{4}} \int_{0}^{x} S(x) \mathrm{d} x+\frac{\lambda}{S^{4}}
$$

and

$$
\lambda=\frac{P_{\text {out }}-P_{\text {in }}-16 v_{w} \int_{0}^{1} \frac{1}{S^{4}(x)}\left[\int_{0}^{x} S(x) \mathrm{d} x\right] \mathrm{d} x}{\int_{0}^{1} \frac{\mathrm{d} x}{S^{4}(x)}} .
$$

$P^{(1)}$ and the velocity components $u^{(1)}$ and $v^{(1)}$ are given by [12]:

$$
P^{(1)}=\left(2 k_{1}+k_{2}\right) \operatorname{Re} \frac{3 r^{2}}{8}\left(\frac{\mathrm{d} P^{(0)}}{\mathrm{d} x}\right)^{2}+f(x),
$$




$$
\begin{aligned}
& u^{(1)}=\frac{\operatorname{Re}}{2304}\left[\frac { \mathrm { d } ^ { 2 } P ^ { ( 0 ) } } { \mathrm { d } x ^ { 2 } } \frac { \mathrm { d } P ^ { ( 0 ) } } { \mathrm { d } x } \left\{2\left(r^{6}-S^{6}\right)\right.\right. \\
& \left.-9 S^{2}\left(r^{4}-S^{4}\right)+18 S^{2}\left(r^{2}-S^{2}\right)\left(24 k_{1}+8 k_{2}+2 S^{2}\right)\right\} \\
& \left.+72\left(\frac{\mathrm{d} P^{(0)}}{\mathrm{d} x}\right)^{2} S \frac{\mathrm{d} S}{\mathrm{~d} x}\left(r^{2}-S^{2}\right)\left(8 k_{1}+4 k_{2}+S^{2}\right)\right] \\
& +\frac{1}{4}\left(r^{2}-S^{2}\right) \frac{\mathrm{d} f(x)}{\mathrm{d} x}, \\
& +\frac{1}{9} r S^{(1)} \frac{\mathrm{d} S}{\mathrm{~d} x} \frac{\mathrm{d} f}{\mathrm{~d} x}-\frac{1}{16}\left(r^{2}-2 S^{2}\right) r \frac{\mathrm{d}^{2} f}{\mathrm{~d} x^{2}}, \\
& \left.+\left(\frac{\mathrm{d}^{2} P^{(0)}}{\mathrm{d} x^{2}}\right)+\frac{\mathrm{d} P^{(0)}}{\mathrm{d} x} \frac{\mathrm{d}^{3} P^{(0)}}{\mathrm{d} x^{3}}\right\} \\
& +\left(r^{6}-4 S^{6}\right)-6 S^{2}\left(r^{4}-3 S^{4}\right) \\
& \left.+36 S\left(r^{2}-4 S^{2}\right)\left(24 k_{1}+8 k_{2}\right)+144 S^{3}\left(r^{2}-3 S^{2}\right)\right\} \\
& +\frac{\mathrm{d}^{2} P^{(0)}}{\mathrm{d} x^{2}} \frac{\mathrm{d} P^{(0)}}{\mathrm{d} x} \frac{\mathrm{d} S}{\mathrm{~d} x}\left\{-24 S^{5}-12 S\left(r^{4}-9 S^{4}\right)\right. \\
& \left.+k^{2}\left(r^{2}-2 S^{2}\right)\left(24 k_{1}+8 k_{2}+2 S^{2}\right)\right\}
\end{aligned}
$$

where

$$
C_{2}=\frac{\left[\frac{64}{\operatorname{Re}}\{f(0)-f(1)\}-\int_{0}^{1} g(x) \mathrm{d} x\right]}{\int_{0}^{1} \frac{\mathrm{d} x}{S^{4}(x)}}
$$

and

$$
\begin{aligned}
& g=\frac{\mathrm{d} P^{(0)}}{\mathrm{d} x} \frac{\mathrm{d}^{2} P^{(0)}}{\mathrm{d} x^{2}} S^{2}\left(3 S^{2}+48 k_{1}+16 k_{2}\right) \\
& +8\left(\frac{\mathrm{d} P^{(0)}}{\mathrm{d} x}\right)^{2} S \frac{\mathrm{d} S}{\mathrm{~d} x}\left(8 k_{1}+4 k_{2}+S^{2}\right) .
\end{aligned}
$$

To obtain the mass and heat transfer, we use the perturbation scheme (27) in (19) and 20). Together with the boundary conditions (24) and (25), and collecting the coefficients of like powers of $\varepsilon$, we get the following set up to first order:

$$
\begin{gathered}
\frac{\partial^{2} \theta^{(0)}}{\partial r^{2}}+\frac{1}{r} \frac{\partial \theta^{(0)}}{\partial r}=-\operatorname{PrEc}\left(\frac{\partial u^{(0)}}{\partial r}\right)^{2}, \\
\frac{\partial^{2} \phi^{(0)}}{\partial r^{2}}+\frac{1}{r} \frac{\partial \phi^{(0)}}{\partial r}=\operatorname{ScSrPrEc}\left(\frac{\partial u^{(0)}}{\partial r}\right)^{2} . \\
\theta^{(0)}=1 \text { and } \phi^{(0)}=1 \text { at } r=S(x), \\
\frac{\partial \theta^{(0)}}{\partial r}=0 \text { and } \frac{\partial \phi^{(0)}}{\partial r}=0 \text { at } r=0 .
\end{gathered}
$$

First order:

$$
\begin{gathered}
\operatorname{Re}\left(u^{(0)} \frac{\partial \theta^{(0)}}{\partial x}+v^{(0)} \frac{\partial \theta^{(0)}}{\partial r}\right)=\frac{1}{\operatorname{Pr}}\left(\frac{\partial^{2} \theta^{(1)}}{\partial r^{2}}+\frac{1}{r} \frac{\partial \theta^{(1)}}{\partial r}\right) \\
+2 \operatorname{Ec} \frac{\partial u^{(0)}}{\partial r} \frac{\partial u^{(1)}}{\partial r}+k_{1} \operatorname{ReEc}\left[\frac { \partial u ^ { ( 0 ) } } { \partial r } \frac { \partial } { \partial r } \left(v^{(0)} \frac{\partial u^{(0)}}{\partial r}\right.\right. \\
\left.\left.+u^{(0)} \frac{\partial u^{(0)}}{\partial x}\right)+2\left(\frac{\partial v^{(0)}}{\partial r}+\frac{\partial u^{(0)}}{\partial x}\right)\left(\frac{\partial u^{(0)}}{\partial r}\right)^{2}\right] \\
+k_{2} \operatorname{ReEc}\left[3\left(\frac{\partial v^{(0)}}{\partial r}+\frac{\partial u^{(0)}}{\partial x}\right)\left(\frac{\partial u^{(0)}}{\partial r}\right)^{2}\right] \\
d\left(u^{(0)} \frac{\partial \phi^{(0)}}{\partial x}+v^{(0)} \frac{\partial \phi^{(0)}}{\partial r}\right)=\frac{\partial^{2} \phi^{(1)}}{\partial r^{2}}+\frac{1}{r} \frac{\partial \phi^{(1)}}{\partial r} \\
+\operatorname{ScSr}\left(\frac{\partial^{2} \theta^{(1)}}{\partial r^{2}}+\frac{1}{r} \frac{\partial \theta^{(1)}}{\partial r}\right) \\
\theta^{(1)}=0 \text { and } \phi^{(1)}=0 \text { at } r=S(x)
\end{gathered}
$$$$
f(x)=f(0)-\frac{\operatorname{Re}}{64} \int_{0}^{x}\left(g+\frac{C_{2}}{S^{4}}\right) \mathrm{d} x,
$$

and 


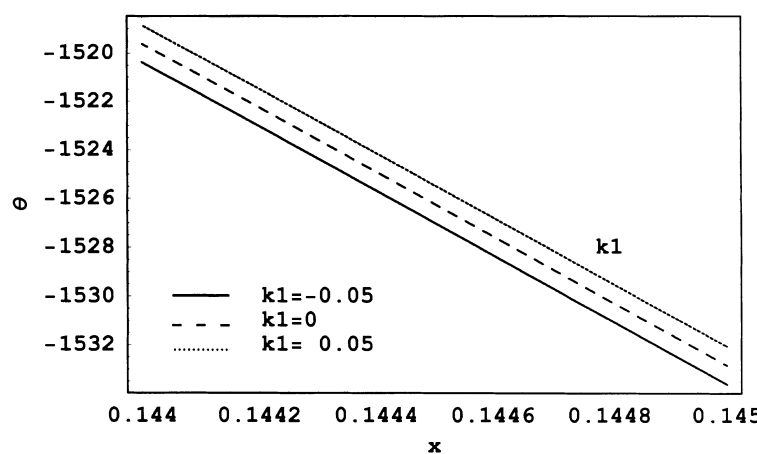

Fig. 1. The temperature distribution is plotted versus $x$, for a system having the particulars $\varepsilon=0.001, P_{\text {out }}=40, P_{\text {in }}$ $=66.66, r=1, S(x)=e^{-x / 2}, k_{2}=10, v_{w}=0.5, \operatorname{Re}=1$, $\mathrm{Ec}=55.5$ and $\mathrm{Pr}=30$ for various values of $k_{1}$. The figure illustrates the relation (50).

$$
\frac{\partial \theta^{(1)}}{\partial r}=0 \text { and } \frac{\partial \phi^{(0)}}{\partial r}=0 \text { at } r=0 .
$$

Equations (39), (40) and the velocity component $u^{(0)}$ along with the conditions (41) and (42) give the temperature $\theta^{(0)}$ and concentration $\phi^{(0)}$ as

$$
\theta^{(0)}=1-\frac{\operatorname{PrEc}}{64}\left(\frac{\mathrm{d} P^{(0)}}{\mathrm{d} x}\right)^{2}\left(r^{4}-S^{4}\right),
$$

and

$$
\phi^{(0)}=1+\operatorname{ScSr} \frac{\operatorname{PrEc}}{64}\left(\frac{\mathrm{d} P^{(0)}}{\mathrm{d} x}\right)^{2}\left(r^{4}-S^{4}\right) .
$$

Equation (43) gives the following solution

$$
\begin{aligned}
& \theta^{(1)}=-\frac{\operatorname{RePr}{ }^{2} \mathrm{Ec}}{128}\left[( \frac { \mathrm { d } P ^ { ( 0 ) } } { \mathrm { d } x } ) ^ { 2 } \frac { \mathrm { d } ^ { 2 } P ^ { ( 0 ) } } { \mathrm { d } x ^ { 2 } } \left(\frac{S^{6} r^{2}}{4}-\frac{r^{4} S^{4}}{16}\right.\right. \\
& \left.+\frac{r^{8}}{128}-\frac{25 S^{8}}{128}\right)+2 \frac{\mathrm{d} S}{\mathrm{~d} x}\left(\frac{\mathrm{d} P^{(0)}}{\mathrm{d} x}\right)^{3}\left(\frac{r^{6} S}{36}-\frac{r^{4} S^{3}}{16}\right. \\
& \left.\left.+\frac{S^{5} r^{2}}{4}-\frac{315^{7}}{144}\right)\right]-\frac{\operatorname{RePrEc}}{192}\left[\left(\frac{\mathrm{d} P^{(0)}}{\mathrm{d} x}\right)^{2} \frac{\mathrm{d}^{2} P^{(0)}}{\mathrm{d} x^{2}}\right. \\
& +\left\{\frac{3 S^{2}}{16}\left(r^{4}-S^{4}\right)\left(24 k_{1}+8 k_{2}+2 S^{2}\right)+\frac{1}{64}\left(r^{8}-S^{8}\right)\right. \\
& \left.-\frac{S^{2}}{12}\left(r^{6}-S^{6}\right)\right\}+\frac{\mathrm{d} S}{\mathrm{~d} x}\left(\frac{\mathrm{d} P^{(0)}}{\mathrm{d} x}\right)^{3} \frac{3}{4} S\left(r^{4}-S^{4}\right)\left(8 k_{1}\right. \\
& \left.\left.+4 k_{2}+S^{2}\right)\right]+\frac{\operatorname{PrEcRe}}{2048}\left(r^{4}-S^{4}\right)\left(g+\frac{C_{2}}{S^{4}}\right) \frac{\mathrm{d} P^{(0)}}{\mathrm{d} x}
\end{aligned}
$$

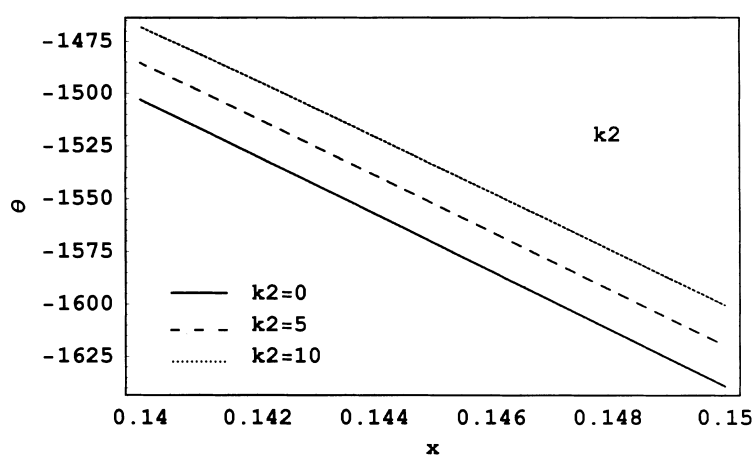

Fig. 2. For the same system as considered in Fig. 1, but when $k_{1}=-0.05$, and for various values of $k_{2}$.

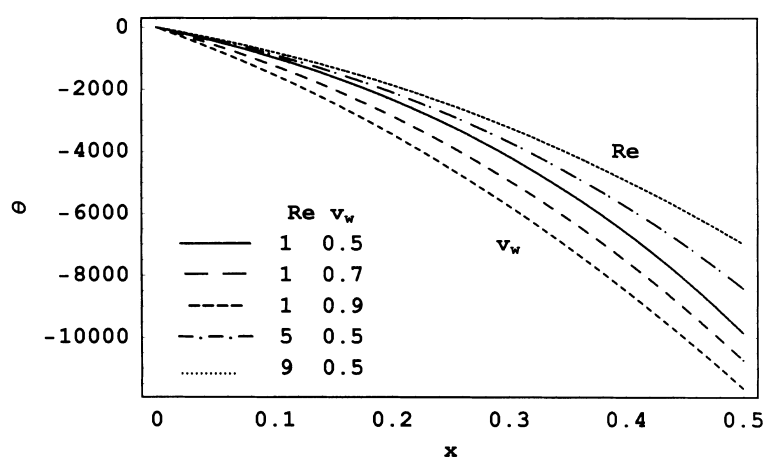

Fig. 3. For the same system as considered in Fig. 2, but when $k_{2}=10$ and for various values of $v_{w}$ and $\mathrm{Re}$.

$$
\begin{gathered}
-k_{1} \operatorname{ReEcPr}\left[\frac{1}{32}\left(\frac{\mathrm{d} P^{(0)}}{d x}\right)^{2} \frac{\mathrm{d}^{2} P^{(0)}}{\mathrm{d} x^{2}}\left(\frac{r^{6}}{12}-\frac{r^{4} S^{2}}{4}+\frac{S^{6}}{6}\right)\right. \\
\left.-\frac{S}{128}\left(r^{4}-S^{4}\right) \frac{\mathrm{d} S}{\mathrm{~d} x}\left(\frac{\mathrm{d} P^{(0)}}{\mathrm{d} x}\right)^{3}\right] \\
-k_{2} \operatorname{Re} \operatorname{EcPr}\left[\frac{3}{64}\left(\frac{\mathrm{d} P^{(0)}}{\mathrm{d} x}\right)^{2} \frac{\mathrm{d}^{2} P^{(0)}}{\mathrm{d} x^{2}}\left(\frac{r^{6}}{36}-\frac{r^{4} S^{2}}{8}+\frac{7 S^{6}}{72}\right)\right. \\
\left.-\frac{3 S}{256}\left(r^{4}-S^{4}\right) \frac{\mathrm{d} S}{\mathrm{~d} x}\left(\frac{\mathrm{d} P^{(0)}}{\mathrm{d} x}\right)^{3}\right] .
\end{gathered}
$$

Therefore the dimensionless temperature distribution become

$$
\begin{aligned}
& \theta=\theta^{(0)}+\varepsilon \theta^{(1)}=1-\frac{\operatorname{PrEc}}{64}\left(\frac{\mathrm{d} P^{(0)}}{\mathrm{d} x}\right)^{2}\left(r^{4}-S^{4}\right) \\
& -\frac{\varepsilon \operatorname{RePr}{ }^{2} \mathrm{Ec}}{128}\left[( \frac { \mathrm { d } P ^ { ( 0 ) } } { \mathrm { d } x } ) ^ { 2 } \frac { \mathrm { d } ^ { 2 } P ^ { ( 0 ) } } { \mathrm { d } x ^ { 2 } } \left(\frac{S^{6} r^{2}}{4}-\frac{r^{4} S^{4}}{16}+\frac{r^{8}}{128}\right.\right.
\end{aligned}
$$




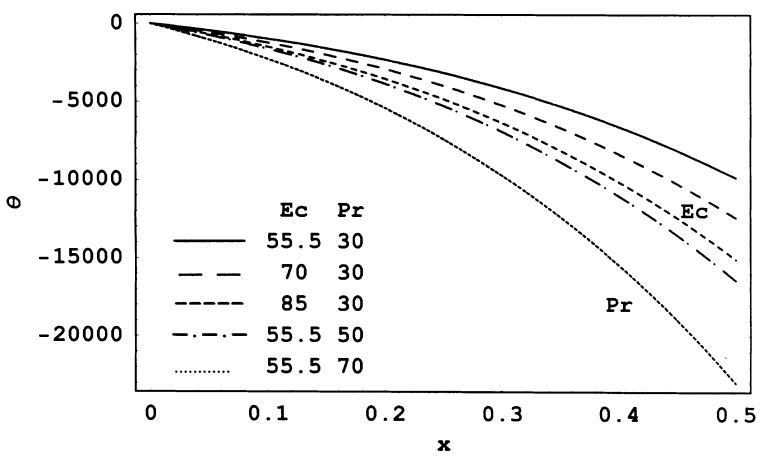

Fig. 4. For the same system as considered in Fig. 3, but when $v_{w}=0.5$ and $\operatorname{Re}=1$ for various values of Ec and Pr.

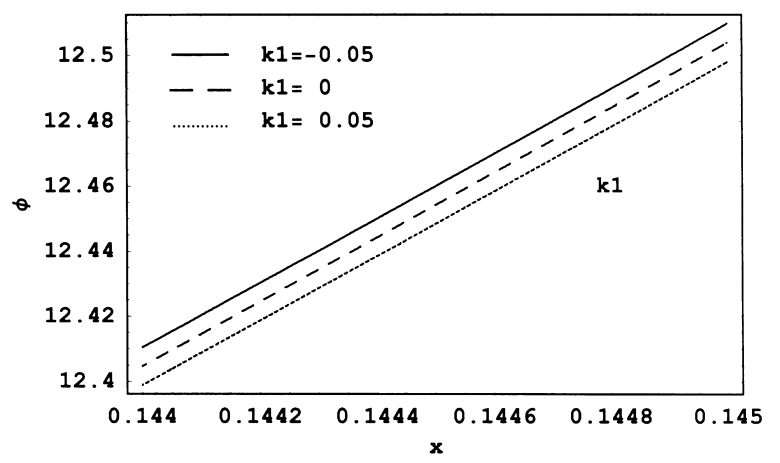

Fig. 5. The concentration distribution is plotted versus $x$, for a system having the particulars $\varepsilon=0.001, P_{\text {out }}=40$, $P_{\text {in }}=66.66, r=1, S(x)=e^{-\frac{x}{2}}, a_{0}=0.7, u_{0}=2, k_{2}=10$, $d=0.9, v_{w}=0.5, \operatorname{Re}=1, \mathrm{Ec}=55.5, \mathrm{Pr}=30, \mathrm{Sc}=0.15$ and $\mathrm{Sr}=0.05$ for various values of $k_{1}$. The figure illustrates the relation (52).

$$
\begin{aligned}
& \left.-\frac{25 S^{8}}{128}\right)+2 \frac{\mathrm{d} S}{\mathrm{~d} x}\left(\frac{\mathrm{d} P^{(0)}}{\mathrm{d} x}\right)^{3}\left(\frac{r^{6} S}{36}-\frac{r^{4} S^{3}}{16}+\frac{S^{5} r^{2}}{4}\right. \\
& \left.\left.-\frac{31 S^{7}}{144}\right)\right]-\frac{\varepsilon \operatorname{RePrEc}}{192}\left[\left(\frac{\mathrm{d} P^{(0)}}{\mathrm{d} x}\right)^{2} \frac{\mathrm{d}^{2} P^{(0)}}{\mathrm{d} x^{2}}\right. \\
& \cdot\left\{\frac{3 S^{2}}{16}\left(r^{4}-S^{4}\right)\left(24 k_{1}+8 k_{2}+2 S^{2}\right)+\frac{1}{64}\left(r^{8}-S^{8}\right)\right. \\
& \left.-\frac{S^{2}}{12}\left(r^{6}-S^{6}\right)\right\}+\frac{\mathrm{d} S}{\mathrm{~d} x}\left(\frac{\mathrm{d} P^{(0)}}{\mathrm{d} x}\right)^{3} \frac{3}{4} S\left(r^{4}-S^{4}\right)\left(8 k_{1}\right. \\
& \left.\left.+4 k_{2}+S^{2}\right)\right]+\frac{\varepsilon \operatorname{PrEcRe}}{2048}\left(r^{4}-S^{4}\right)\left(g+\frac{C_{2}}{S^{4}}\right) \frac{\mathrm{d} P^{(0)}}{\mathrm{d} x} \\
& -\varepsilon k_{1} \operatorname{ReEcPr}\left[\frac{1}{32}\left(\frac{\mathrm{d} P^{(0)}}{\mathrm{d} x}\right)^{2} \frac{\mathrm{d}^{2} P^{(0)}}{\mathrm{d} x^{2}}\left(\frac{r^{6}}{12}-\frac{r^{4} S^{2}}{4}+\frac{S^{6}}{6}\right)\right.
\end{aligned}
$$

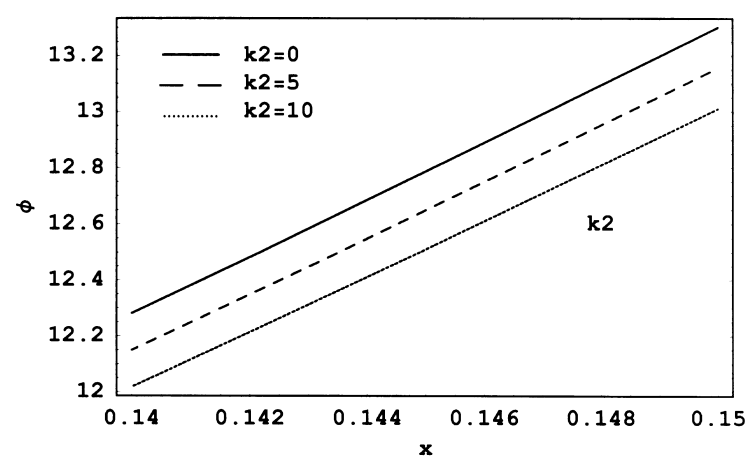

Fig. 6. For the same system as considered in Fig. 5, but when $k_{1}=-0.05$, and for various values of $k_{2}$.

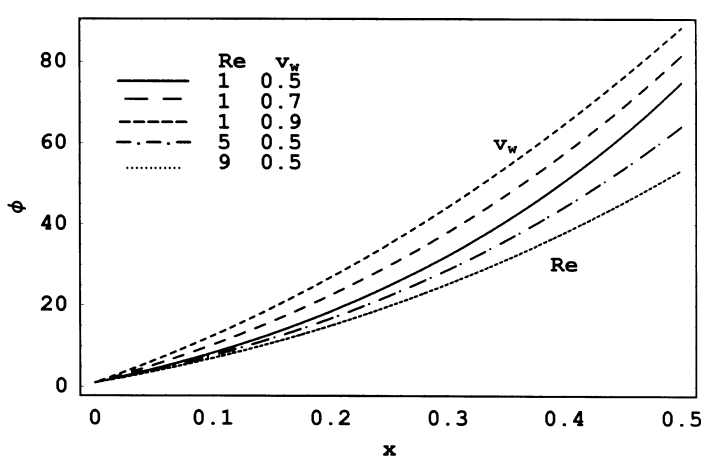

Fig. 7. For the same system as considered in Fig. 6, but when $k_{2}=10$, and for various values of $v_{w}$ and Re.

$$
\left.-\frac{S}{128}\left(r^{4}-S^{4}\right) \frac{\mathrm{d} S}{\mathrm{~d} x}\left(\frac{\mathrm{d} P^{(0)}}{\mathrm{d} x}\right)^{3}\right]
$$

$$
\begin{array}{r}
-\varepsilon k_{2} \operatorname{ReEcPr}\left[\frac{3}{64}\left(\frac{\mathrm{d} P^{(0)}}{\mathrm{d} x}\right)^{2} \frac{\mathrm{d}^{2} P^{(0)}}{\mathrm{d} x^{2}}\left(\frac{r^{6}}{36}-\frac{r^{4} S^{2}}{8}+\frac{7 S^{6}}{72}\right)\right. \\
\left.-\frac{3 S}{256}\left(r^{4}-S^{4}\right) \frac{\mathrm{d} S}{\mathrm{~d} x}\left(\frac{\mathrm{d} P^{(0)}}{\mathrm{d} x}\right)^{3}\right] .
\end{array}
$$

Equation (44) gives the solution

$$
\begin{gathered}
\phi^{(1)}=d \frac{\mathrm{ScSrPrEc}}{256}\left[\frac { 1 } { 2 } ( \frac { \mathrm { d } P ^ { ( 0 ) } } { \mathrm { d } x } ) ^ { 2 } \frac { \mathrm { d } ^ { 2 } P ^ { ( 0 ) } } { \mathrm { d } x ^ { 2 } } \left(\frac{r^{8}}{32}-\frac{S^{4} r^{4}}{4}\right.\right. \\
\left.+S^{6} r^{2}-\frac{25 S^{8}}{32}\right)+\frac{\mathrm{d} S}{\mathrm{~d} x}\left(\frac{\mathrm{d} P^{(0)}}{\mathrm{d} x}\right)^{3}\left(\frac{r^{6} S}{9}-\frac{r^{4} S^{3}}{4}\right. \\
\left.\left.+r^{2} S^{5}-\frac{31}{36} S^{7}\right)\right]-\operatorname{ScSr} \theta^{(1)}
\end{gathered}
$$




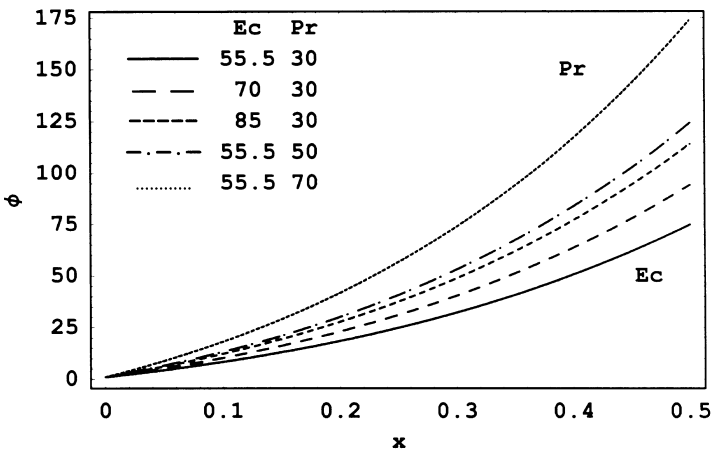

Fig. 8. For the same system as considered in Fig. 7, but when $\operatorname{Re}=1, \mathrm{v}_{\mathrm{w}}=0.5$, and for various values of Pr and Ec.

Therefore the nondimensional concentration distribution becomes

$$
\begin{gathered}
\phi=\phi^{(0)}+\varepsilon \phi^{(1)}=1+\operatorname{ScSr} \frac{\operatorname{PrEc}}{64}\left(\frac{\mathrm{d} P^{(0)}}{\mathrm{d} x}\right)^{2}\left(r^{4}-S^{4}\right) \\
+\varepsilon \mathrm{d} \frac{\mathrm{ScSrPrEc}}{256}\left[\frac { 1 } { 2 } ( \frac { \mathrm { d } P ^ { ( 0 ) } } { \mathrm { d } x } ) ^ { 2 } \frac { \mathrm { d } ^ { 2 } P ^ { ( 0 ) } } { \mathrm { d } x ^ { 2 } } \left(\frac{r^{8}}{32}-\frac{r^{4} S^{4}}{4}\right.\right. \\
\left.+S^{6} r^{2}-\frac{25 S^{8}}{32}\right)+\frac{\mathrm{d} S}{\mathrm{~d} x}\left(\frac{\mathrm{d} P^{(0)}}{\mathrm{d} x}\right)^{3}\left(\frac{r^{6} S}{9}-\frac{r^{4} S^{3}}{4}\right. \\
\left.\left.+r^{2} S^{5}-\frac{31 S^{7}}{36}\right)\right]-\varepsilon \operatorname{ScSr} \theta^{(1)} .
\end{gathered}
$$

\section{Heat Flux}

From the temperature field equation (50) we can now study the rate of heat transfer (heat flux). This rate is given by [13]

$$
q_{w}=-\left.k \frac{\partial T}{\partial R}\right|_{R=a(X)} .
$$

By using (15), the dimensionless heat flux may be expressed by

$$
\mathrm{Nu}_{x}=\frac{a_{0} q_{w}}{k T_{1}}
$$

where $\mathrm{Nu}_{x}$ is the Nusselt number.

From (50) and (54), $\mathrm{Nu}_{x}$ becomes

$$
\mathrm{Nu}_{x}=-\left.\frac{\partial \theta}{\partial r}\right|_{r=S(x)}
$$

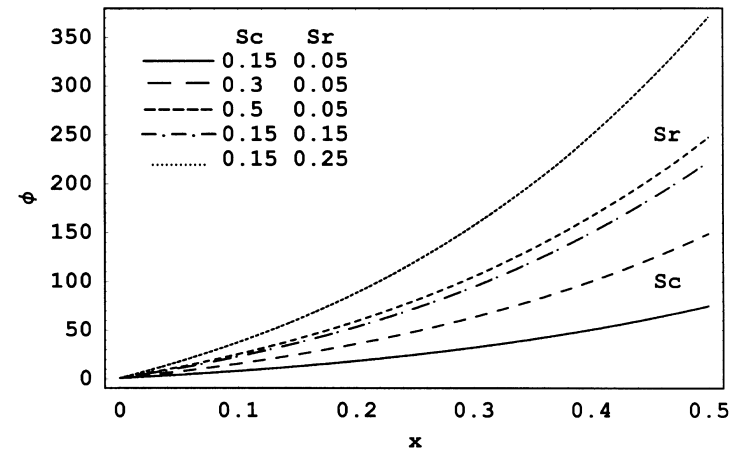

Fig. 9. For the same system as considered in Fig. 8, but when $\operatorname{Pr}=30, \mathrm{Ec}=55.5$ and for various values of $\mathrm{Sr}$ and $\mathrm{Sc}$.

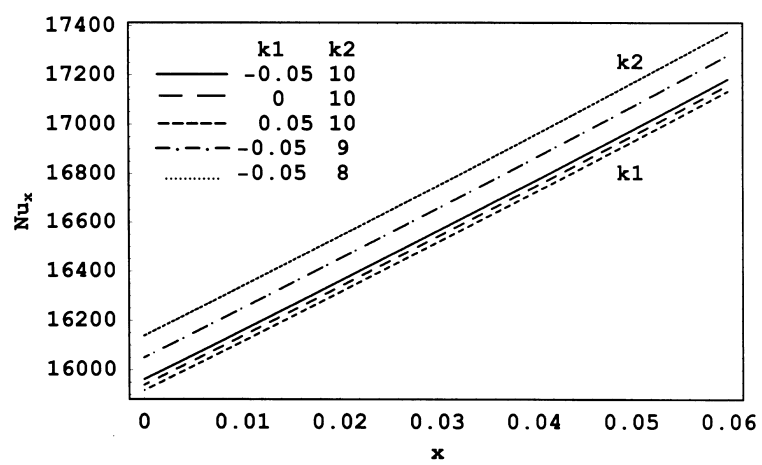

Fig. 10. The Nusselt number is plotted versus $x$, for a system having the particulars $\varepsilon=0.001, r=1, P_{\text {out }}=40, P_{\text {in }}=$ 66.66, $S(x)=e^{-\frac{x}{2}}, v_{w}=0.5, \operatorname{Re}=1, E c=55.5, \operatorname{Pr}=30$, and for various values of $k_{1}$ and $k_{2}$. The figure illustrates the relation (56).

$$
=-\left.\frac{\partial \theta^{(0)}}{\partial r}\right|_{r=S(x)}-\left.\varepsilon \frac{\partial \theta^{(1)}}{\partial r}\right|_{r=S(x)}+o\left(\varepsilon^{2}\right),
$$

or

$$
\mathrm{Nu}_{x}=\frac{\operatorname{PrEc}}{16} S^{3}\left(\frac{\mathrm{d} P^{(0)}}{\mathrm{d} x}\right)^{2}+\varepsilon \frac{\operatorname{RePrEc}}{192}
$$

$\cdot\left[\frac{3}{8} \frac{C_{2}}{S}+\left(\frac{\mathrm{d} P^{(0)}}{d x}\right)^{2} \frac{d^{2} P^{(0)}}{\mathrm{d} x^{2}}\right.$

$$
\cdot\left\{3 S^{5}\left(11 k_{1}+3 k_{2}\right)+\frac{3}{4} S^{7}\left(3+\frac{5}{8} \operatorname{Pr}\right)\right\}
$$

$$
\left.+\frac{\mathrm{d} S}{\mathrm{~d} x}\left(\frac{\mathrm{d} P^{(0)}}{\mathrm{d} x}\right)^{3}\left\{3 S^{4}\left(14 k_{1}+4 k_{2}+2 S^{2}\right)+\frac{5}{4} S^{6} \operatorname{Pr}\right\}\right] .
$$




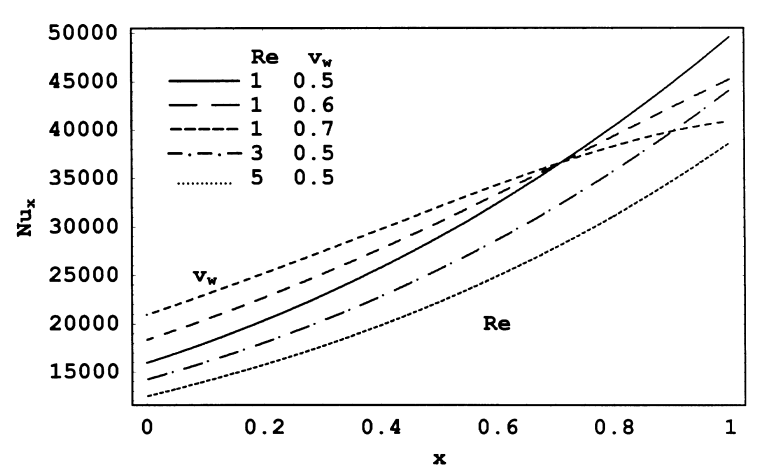

Fig. 11. For the same system as considered in Fig. 10, but when $k_{1}=-0.05, k_{2}=10$, and for various values of $v_{w}$ and $R e$.

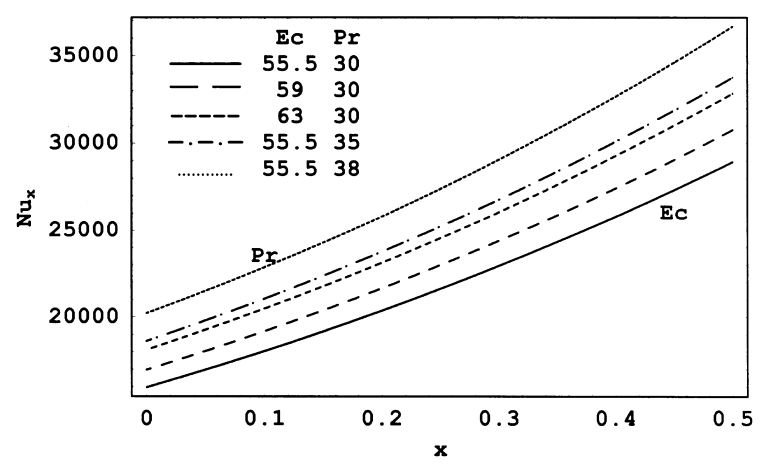

Fig. 12. For the same system as considered in Fig. 11, but when $v_{w}=0.5, \operatorname{Re}=1$, and for various values of Ec and Pr.

\section{Diffusion Rate}

From the concentration field equation (52) we can determine the rate of mass transfer (diffusion rate). This rate is given by [13]

$$
h_{m}=-\left.D \frac{\partial C}{\partial R}\right|_{R=a(X)}
$$

Then

$$
\mathrm{Nm}_{x}=\frac{h_{m} a_{0}}{D C_{0}},
$$

where $\mathrm{Nm}_{x}$ is the Sherwood number.

From (52) and (58), one gets

$$
\begin{aligned}
\mathrm{Nm}_{x} & =-\left.\frac{\partial \phi}{\partial r}\right|_{r=S(x)} \\
& =-\left.\frac{\partial \phi^{(0)}}{\partial r}\right|_{r=S(x)}-\left.\varepsilon \frac{\partial \phi^{(1)}}{\partial r}\right|_{r=S(x)}+o\left(\varepsilon^{2}\right)
\end{aligned}
$$

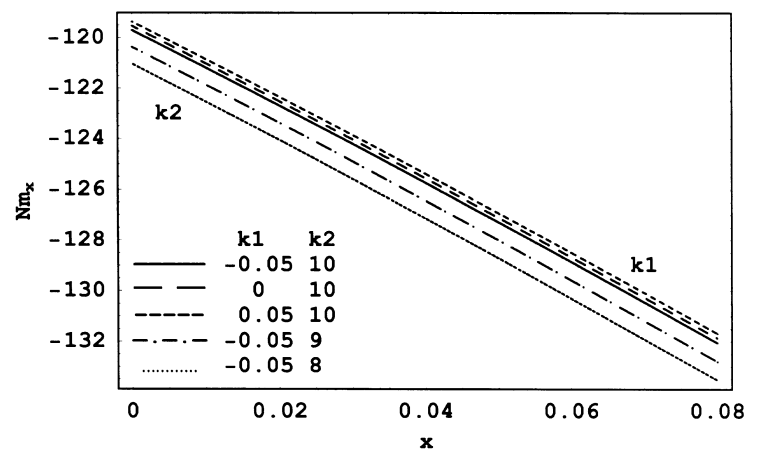

Fig. 13. The Sherwood number is plotted versus $x$, for a system having the particulars $\varepsilon=0.001, r=1, P_{\text {out }}=40$, $P_{\text {in }}=66.66, S(x)=e^{-x / 2}, a_{0}=0.7, u_{0}=2, v_{w}=0.5$, $R e=1, \mathrm{Ec}=55.5, \operatorname{Pr}=30, d=0.9, \mathrm{Sc}=0.15, \mathrm{Sr}=0.05$, and for various values of $k_{1}$ and $k_{2}$. The figure illustrates the relation (60).

or

$$
\begin{aligned}
& \mathrm{Nm}_{x}=-\mathrm{ScSrNu}_{x}-\varepsilon \frac{d \mathrm{ScSrPrEc}}{256} \\
& \cdot\left[\frac{5}{8} S^{7}\left(\frac{\mathrm{d} P^{(0)}}{\mathrm{d} x}\right)^{2} \frac{\mathrm{d}^{2} P^{(0)}}{\mathrm{d} x^{2}}+\frac{5}{3} S^{6} \frac{\mathrm{d} S}{\mathrm{~d} x}\left(\frac{\mathrm{d} P^{(0)}}{\mathrm{d} x}\right)^{3}\right] .
\end{aligned}
$$

\section{Results and Discussion}

Now, a numerical discussion will be made to show the effects of the parameters entering the problem on the temperature $\theta$, the concentration $\phi$, and the Nusselt and Sherwood numbers $\mathrm{Nu}_{x}$ and $\mathrm{Nm}_{x}$, respectively. Therefore (50), (52), (56) and (60) are evaluated by setting $\varepsilon=0.001, P_{\text {out }}=40$ and $P_{\text {in }}=66.66$ for a convergent tube $S(x)=e^{-x / 2}$.

The results are shown in Figures 1 - 17. The effects of non-Newtonian parameters, the suction parameter, Eckert number, Prandtl number and Reynolds number on the temperature distribution in a convergent tube are shown in Figs. 1 - 6. The expression (50) is evaluated by taking $r=1$, and the values $\theta$ are plotted versus $x$.

In Figs. 1 and 2 it is observed that the temperature distribution increases as $k_{1}$ and $k_{2}$ increase. In Fig. 3, we observe that $\theta$ increases as Re increases while it decreases with the increase of $v_{w}$.

In Fig. 4 it is found that $\theta$ decreases with the increase of Ec and Pr.

Figures 5 - 9 are graphed to illustrate the effects of different parameters on the concentration distribution 


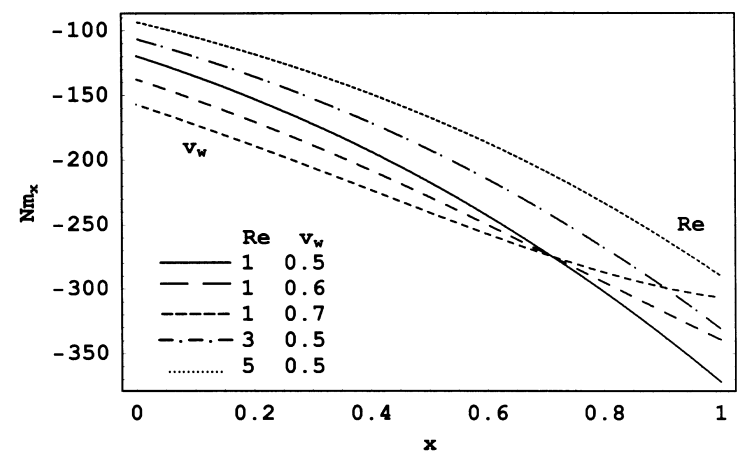

Fig. 14. For the same system as considered in Fig. 13, but when $k_{1}=-0.05, k_{2}=10$, and for various values of $v_{w}$ and Re.

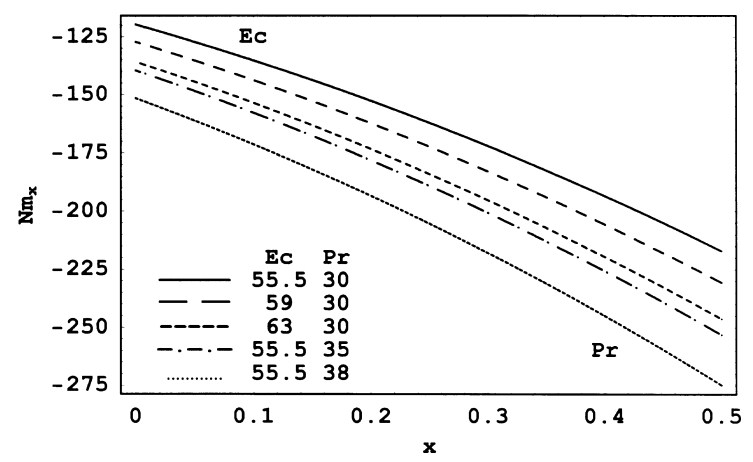

Fig. 15. For the same system as considered in Fig. 14, but when $v_{w}=0.5, \operatorname{Re}=1$, and for various values of Ec and Pr.

$\phi$. Therefore the $(\phi-x)$ plans are graphed according (52). In Figs. 5, 6 and 7 it is found that $\phi$ decreases with increase of $k_{1}, k_{2}$ and Re, while it increases as $v_{w}$ increases. In Figs. 8 and 9 it is observed that $\phi$ increases as Pr, Ec, Sr and Sc increase. From the study of graphs it is found that the effects of various parameters entering into the problem on $\theta$ and $\phi$ similary for the divergent tube at $S(x)=e^{x / 2} \cdot \theta$ increases as the parameters increase while $\phi$ decreases with the increase of the different parameters. The values of $\mathrm{Nu}_{x}$ and $\mathrm{Nm}_{x}$ are plotted versus $x$ in Figures $10-17$.

In Fig. 10 it is observed that $\mathrm{Nu}_{x}$ decreases with the increase of non-Newtonian parameters. $\mathrm{Nu}_{x}$ de-

[1] M. N. Mathur and R. K. Bhatnagar, ZAMM 47, 379 (1967).

[2] M. E. O’Neill, Chem. Eng. Sci. 25, 283 (1970).

[3] A. C. Srivastava, J. Fluid Mech. 17, 171 (1963).

[4] Y. Kawase and J. J. Ulbrecht, Cand. J. Chem. Eng. 61, 791 (1983).

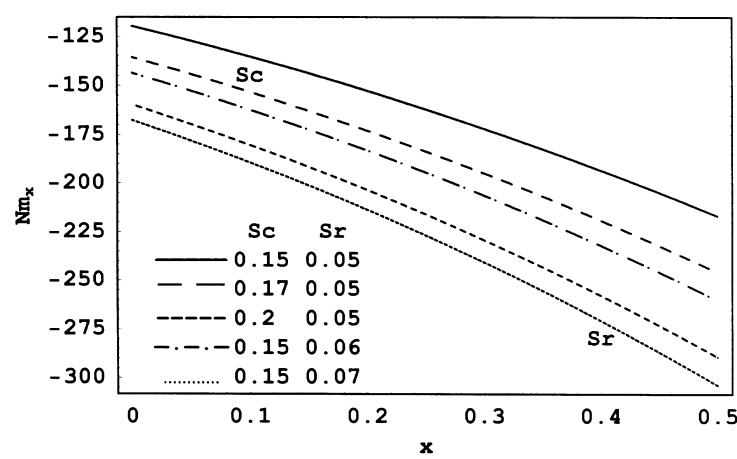

Fig. 16. For the same system as considered in Fig. 15, but when $\mathrm{Ec}=55.5, \operatorname{Pr}=30$ and for various values of $\mathrm{Sc}$ and $\mathrm{Sr}$.

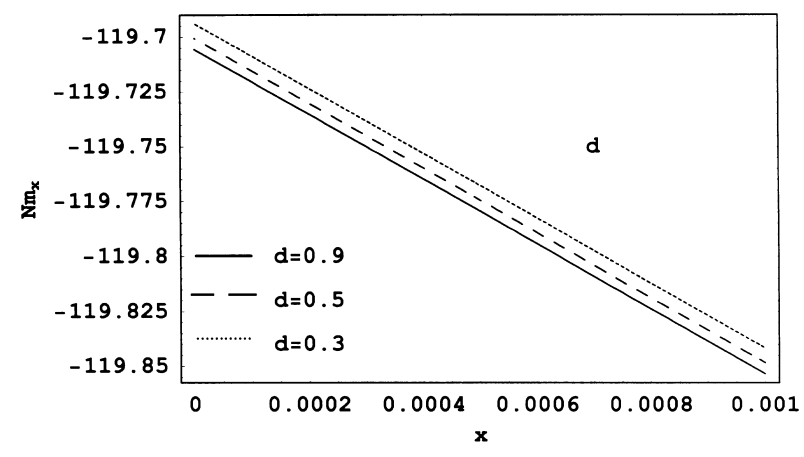

Fig. 17. For the same system as considered in Fig. 16, but when $\mathrm{Sc}=0.15, \mathrm{Sr}=0.05$, and for various values of $d$.

creases with increases of Re. This effects is indicated in Figure 11. In Fig. 11 we observed that $\mathrm{Nu}_{x}$ increases as $v_{w}$ increases, but at $x=0.72, \mathrm{Nu}_{x}$ starts to decrease with increasing $v_{w}$ (the suction parameter). In Fig. 12 it is found that $\mathrm{Nu}_{x}$ increases as $\mathrm{Ec}$ and $\mathrm{Pr}$ increase.

In Figs. 13 and 14 we observed that $\mathrm{Nm}_{x}$ increases as $k_{1}, k_{2}$ and $\mathrm{Re}$ increase, while $\mathrm{Nm}_{x}$ decreases with increasing $v_{w}$. At $x=0.72, N m_{x}$ start increasing with the increasing $v_{w}$.

Figures 15, 16 and 17 show that $\mathrm{Nm}_{x}$ decreases with increase of the parameters $\mathrm{Pr}, \mathrm{Ec}, \mathrm{Sc}, \mathrm{Sr}$ and $d$.

[5] D. C. Dalal and B. S. Mazumder, Inter. J. Nonlinear Mech. 33, 135 (1998).

[6] E. C. Pereira, M. Bhattacharya, and R. V. Morey, Trans. ASAE 32, 256 (1989).

[7] I. C. Hung and Y. Perng, Int. J. Heat Fluid Flow 12, 263 (1991). 
[8] R. S. Parikh and R. Mahalingam, Int. Commun. Heat Mass Transfer. 15, 1 (1988).

[9] Peeyush Chandra and J. S. V. R. Krishna Prasad, J. Math. Phys. Sci. 26, 19 (1992).

[10] M. E. S. Ahmed and H. A. Attia, Can. J. Phys. 76, 391 (1998).
[11] V. N. Kurdyumov and A. Linan, J. Fluid Mech. 427, 389 (2001).

[12] J. S. V. R. Krishna Prasad and Peeyush Chandra, Eur. J. Mech. B/Fluids 11, 277 (1992).

[13] N. G. Kafoussias and E. W. Williams Int. J. Engng, Sci. 33, 1369 (1995). 